\title{
COMUNICACIÓN
}

\section{Eficácia do diazinon em uma formulação de uso otológico no tratamento da sarna otodécica em cães}

\author{
CLARISSA PIMENTEL DE SOUZA*, GUILHERME GOMES VEROCAI*, THAÍS RIBEIRO CORREIA*, \\ RAQUEL MOREIRA PIRES DOS SANTOS MELO*, \\ MAÍRA CRUZ HOLANDA CAVALCANTI**, e FABIO BARBOUR SCOTT***
}

\section{EFFICACY OF DIAZINON IN AN OTIC SOLUTION FOR THE TREATMENT OF OTODECTIC MANGE ON DOGS}

Diazinon is an organophospate used on the ectoparasite control especially in production animals. The objective of this study was to evaluate the efficacy of an otic solution containing this insecticide on the treatment of Otodectes cynotis infestations in dogs. Ten naturally infested beagle dogs were examined by bilateral otoscopy three days before treatment and on the treatment day. The dogs were randomly divided into two groups, which five were the control group and the other five treated. On the treated group $1 \mathrm{ml}$ of the otic solution was applied in each ear for seven following days, corresponding to $10 \mathrm{mg}$ of diazinon per ear everyday. All animals were examined by otoscopy on days $+1,+4,+10,+17+24 e+31$ after treatment. The control dogs showed mites during the whole period of observation. On the treated group the otic solution had a $100 \%$ efficacy on each day of observation until day +31 .

Key words: Otodectes cynotis, diazinon, dogs.

\section{INTRODUÇÃO}

O ácaro Otodectes cynotis habita o conduto auditivo de várias espécies animais, principalmente cães e gatos, sendo a infestação denominada sarna otodécica ${ }^{1}$. A transmissão ocorre por contato direto, sendo os ácaros altamente contagiosos. O ciclo de vida se passa todo sobre os hospedeiros, durando em torno de três semanas ${ }^{2}$. É um parasito bastante ativo e sua presença está, geralmente, associada a prurido e ao aumento na produção de secreção, que pode gerar infecções secundárias bacterianas e fúngicas e causar grande incômodo aos animais. O diagnóstico, em geral, é feito através de otoscopia ou visualização do cerumem sob microscópio esterioscópico ${ }^{3}$.

Em outros países e inclusive no Brasil, vários produtos antiparasitários vêm sendo utilizados para o tratamento da infestação por $O$. cynotis em diferentes espécies animais como: ivermectina $^{4}$, selamectina ${ }^{5}$, imidacloprid ${ }^{6}$, fipronil ${ }^{7}$, d-fenotrina ${ }^{8}$ e tiabendazol $^{9}$, embora algumas vezes não contenham tal indicação em suas bulas.

* Curso de Pós-graduação em Ciências Veterinárias - Instituto de Veterinária / Universidade Federal Rural do Rio de Janeiro.

** Curso de Graduação em Medicina Veterinária - Universidade Federal Rural do Rio de Janeiro.

*** Departamento de Parasitologia Animal - Instituto de Veterinária / Universidade Federal Rural do Rio de Janeiro. 
A solução otológica que contém pimaricina, neomicina, acetato de dexametasona e diazinon a $1 \%$ (Natalene $^{\circledR}$ - Virbac Saúde Animal) é um dos poucos produtos disponíveis no mercado veterinário brasileiro indicado para o tratamento da sarna otodécica.

$\mathrm{O}$ diazinon é um inseticida pertencente à classe dos organofosforados, muito utilizado no controle de ectoparasitos, principalmente em animais de produção. Sua ação ocorre através da inibição da enzima colinesterase, levando o parasito à paralisia e morte ${ }^{10}$.

O objetivo deste estudo foi avaliar a eficácia do diazinon contido em solução otológica no tratamento de infestações por $O$. cynotis em cães.

\section{MATERIAL E MÉTODOS}

Foram utilizados 10 cães da raça beagle pertencentes ao Canil de Experimentação do Laboratório de Desenvolvimento de Produtos Parasiticidas situado no Convênio Embrapa Sanidade Animal da Universidade Federal Rural do Rio de Janeiro, localizado no município de Seropédica, RJ, Brasil. Todos os animais apresentavam infestação natural pelo ácaro, diagnosticada através de otoscopia bilateral realizada com auxílio de um otoscópio com espéculos veterinários (Gowlands ${ }^{\circledR}$ ). Cada orelha de cada cão foi considerada uma unidade experimental, pois são independentes. Os cães foram examinados três dias antes do tratamento (dia -3) e no dia do tratamento (dia 0). Foram divididos aleatoriamente em dois grupos, sendo que cinco cães foram utilizados como grupo controle, não recebendo tratamento e, os demais tratados. Após o tratamento os animais dos grupos controle e tratado foram mantidos em canis separados. Nos animais pertencentes ao grupo tratado foi aplicado em cada conduto auditivo, durante sete dias consecutivos, $1 \mathrm{ml}$ da solução otológica contendo pimaricina, neomicina, acetato de dexametasona e diazinon, correspondendo a $10 \mathrm{mg}$ de diazinon por orelha. Não foi realizada limpeza dos condutos auditivos com retirada de secreção otológica durante a experimentação. Todos os animais foram reexaminados através de otoscopia nos dias +1 , $+3,+10,+17+24$ e +31 após o tratamento. Nenhum tipo de controle ambiental foi efetuado. A análise estatística dos resultados foi feita através do teste de $\chi^{2}$ corrigido (Yates) ${ }^{11}$.

\section{RESULTADOS E DISCUSSÃO}

Os cães pertencentes ao grupo controle apresentaram ácaros $O$. cynotis em ambas as orelhas, em todos os dias de observação. Já os animais tratados, não apresentaram ácaros a partir do primeiro dia de tratamento até o final da observação. No grupo tratado a eficácia média do produto foi de $100 \%$ em todos os dias de observação após o tratamento (Tabela 1) Não foram observadas reações adversas consideradas relacionadas ao tratamento durante o período de experimentação. Analisando estatisticamente os resultados encontrados através do teste de $C^{2}$ corrigido (Yates) obteve-se o valor 16,20, que é significativo mesmo com o número de orelhas utilizado, pois os resultados estão bem determinados.

Não foram encontradas citações na literatura consultada sobre o uso do diazinon para o tratamento de infestações por $O$. cynotis em condutos auditivos de cães. Em 1992, um pesquisador utilizou uma solução de diazinon a 4\% para o tratamento de sarna otodécica em raposas e obtiveram ótimos resultados ${ }^{12}$. Este inseticida vem sendo empregado com sucesso

Tabela 1. Presença do ácaro Otodectes cynotis nas orelhas direita e esquerda dos cães pertencentes ao grupo tratado com Natalene ${ }^{\circledR}$, antes e após o tratamento

\begin{tabular}{|c|c|c|c|c|c|c|c|c|c|c|c|}
\hline od & Dia +1 & Dia +3 & Dia + & & Dia & & Dia & & Dia & & Dia +31 \\
\hline & 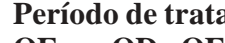 & nento dos a & nimais & & & & & & & & \\
\hline OD & OD OE & OD $\quad \mathrm{OE}$ & OD & OE & OD & OE & OD & $\mathrm{OE}$ & OD & $\mathrm{OE}$ & OD \\
\hline
\end{tabular}

\begin{tabular}{|c|c|c|c|c|c|c|c|c|c|c|c|c|c|c|c|c|}
\hline 1 & + & + & - & - & - & - & - & - & - & - & - & - & $=$ & - & - & - \\
\hline 2 & + & + & - & - & - & - & - & - & - & - & - & - & - & - & - & - \\
\hline 3 & + & + & - & - & - & - & - & - & - & - & - & - & - & - & - & - \\
\hline 4 & + & + & - & - & - & - & - & - & - & - & - & - & - & - & - & - \\
\hline 5 & + & + & - & - & - & - & - & - & - & - & - & - & - & - & - & - \\
\hline
\end{tabular}


em diferentes formulações no tratamento de outras parasitoses de animais de companhia, como escabiose e infestações por pulgas e carrapato $^{13-16}$. O diazinon também vem sendo utilizado no tratamento de sarnas causadas por Psoroptes em ovinos ${ }^{17}$ e em sarnas de orelha por $P$. equi em coelhos, onde se observa alta eficácia deste inseticida, podendo ser usado inclusive em ninhadas de animais logo após o nascimento ${ }^{18}$.

A associação do diazinon aos antimicrobianos e antiinflamatório é útil, pois as infestações por O. cynotis estão na maioria das vezes associadas a hiperemia das orelhas, que pode resultar em inflamação e proliferação de microrganismos, causando infecções secundárias ${ }^{19}$. Sendo assim, esta combinação confere maior praticidade ao produto, uma vez que o proprietário não precisa dispor de diferentes medicamentos para o tratamento de seus animais com otite parasitária, podendo lançar mão apenas da solução otológica polivalente testada.

O diazinon aplicado uma vez ao dia na dose de $10 \mathrm{mg}$ por conduto auditivo durante um período de 7 dias foi eficaz contra otites parasitárias por $O$. cynotis em cães.

\section{RESUMO}

O objetivo deste estudo foi avaliar a eficácia de uma solução otológica contendo o inseticida diazinon a $1 \%$ no tratamento de infestações por Otodectes cynotis em cães. Foram utilizados 10 cães da raça beagle infestados naturalmente, sendo o ácaro diagnosticado através de otoscopia bilateral. Os animais foram divididos em dois grupos iguais, um controle e o outro tratado. No tratado foi aplicado $1 \mathrm{ml}$ da solução otológica em cada conduto auditivo, durante sete dias consecutivos, correspondendo a 10 $\mathrm{mg}$ de diazinon por orelha por dia. Todos os animais foram examinados através de otoscopia nos dias $+1,+4,+10,+17+24$ e +31 após o tratamento. Os cães controle apresentavam ácaros em todos os dias de observação. No grupo tratado, o percentual de eficácia do produto foi de $100 \%$ em todos os dias de observação, até o dia +31 .

\section{REFERÊNCIAS}

1.- SWEATMAN G K. Biology of Otodectes cynotis, the ear canker mite of carnivores. Can J Zool 1958; 36: 849-62.
2.- SCOTT D W, MILLER W H, GRIFFIN C E. Muller \& Kirk's Small Animal Dermatology. $6^{\text {th }}$ edition. W. B. Saunders Company, Philadelphia, 2001, 1528 p.

3.- SOUZA C P, SCOTT F B, PEREIRA M J S. Validade e reprodutibilidade da otoscopia e do reflexo otopodal no diagnótico da infestação por Otodectes cynotis em cães. Rev Bras Parasitol Vet 2004; 13: 111-4.

4.- HUANG H P, LIEN Y H. Otic ivermectin in the treatment of feline Otodectes infestation. Vet Dermatol 2000; 11: 41.

5.- SIX R H, CLEMENCE R G, THOMAS C A, et al. Efficacy and safety of selamectin against Sarcoptes scabiei on dogs and Otodectes cynotis on dogs and cats presented as veterinary patients. Vet Parasitol 2000; 91: 291-309.

6.- LUCAS R, JORGE F Z, SHIGUEMOTO L. Uso do imidacloprid no tratamento de otoacaríase em animais domésticos. A Hora Vet 2003; 23: 11-5.

7.- SOUZA C P, CORREIA T R, FERNANDES J I, et al. Eficácia do fipronil no tratamento da sarna otodécica em cães. Rev Univ Rural 2004; 24 (supl): 23-4.

8.- SCOTT F B, RODRIGUES M C D, AZEVEDO S C S, et al. Eficácia acaricida da d-fenotrina $\left(\right.$ Mypet $\left.^{\circledR}\right)$ no tratamento das sarnas otodécica e sarcóptica em cães. A Hora Vet 2005; 25: 37-41.

9.- VEROCAI G G, SOUZA C P, CORREIA T R, et al. Eficácia do tiabendazol no tratamento da sarna otodécica em cães. Rev Bras Parasitol Vet. 2004; 13 (supl 1): 344.

10.- BOWMAN D. Georgi‘s Parasitology for Veterinarians. $8^{\text {th }}$ edition. Saunders, St Louis, 2003; 422 p.

11.- SAMPAIO I B M. Estatística Aplicada à Experimentação Animal.1 ${ }^{\mathrm{a}}$.edição. Editora Fundação de Ensino e Pesquisa em Medicina Veterinária e Zootecnia, Belo Horizonte, 1998; 265 p.

12.- PACIEJEWSKI S. Control of scabies in breeding foxes. Med Wet 1992; 48: 506-8.

13.- ALCAINO H, GORMAN T, BUDROVIC M T. Evaluation of the efficacy of some acaricides against Rhipicephalus sanguineus. Parasitol al Día 1991; 15 ; 15-20.

14.- KAMBOJ D S, SINGH K B, SINGH R P et al. Studies on the therapeutic efficacy of diazinon against canine scabies. Ind Vet J 1995; 72 :769-70.

15.- ONISHI T, INOKUMA H, UZUKA Y. Effects of diazinon $20 \%$ spot on on the flea and tick infestation in dogs and cats. J Jap Vet Med Assoc 1996; 49: 6414.

16.- FRANC M, CADIERGUES M C. Comparative activity in dogs of deltamethrin and diazinon impregnated collars against Ctenocephalides felis. Am J Vet Res 1998; 59: 59-60.

17.- BATES P G. Epidemiology of subclinical ovine psoroptic otoacariasis in Great Britain. Vet Rec 1996; 138: 388-93.

18.- DONKÓ T. Comparative study of rabbit ear mange treatment and epizootic aspects in medicinal prevention. Acta Agraria Kaposvariensis 1999; 3: 41-9.

19.- SOUZA C P. Otite parasitária por Otodectes cynotis (Hering, 1838) (Acari: Psoroptidae): diagnóstico, aspectos epidemiológicos e clínicos. Dissertação de Mestrado. Departamento de Parasitologia Veterinária, UFRRJ, 2004. 